ISSN: 0213-2060

DOI: https://doi.org/10.14201/shhme2021391123146

\title{
LE POLITICHE URBANISTICHE ED EDILIZIE DELLE CITTÀ ITALIANE NEGLI STATUTI DEI SECOLI XII E XIII
}

\author{
The Italian Cities' Urban Planning and Building Policies in the Statutes of the Twelfth \\ and Thirteenth Centuries
}

\begin{abstract}
Federico LATTANZIO
Dipartimento di Storia, Patrimonio Culturale, Formazione e Societá. Universitá degli Studi di Roma Due Tor Vergata.Via Pietro Benedetti 20, 00126 Roma (Italia). C.e: federico-83@hotmail.it; ORCID: https:/lorcid. org/0000-0003-3950-7928
\end{abstract}

Recibido: 2021-02-17

Revisado: 2021-03-20

Aceptado: 2021-04-03

RESUMEN: Este artículo analiza las políticas de planificación y urbanización de las ciudades italianas mediante el estudio de los estatutos de los siglos XII y XIII. El texto se enfrenta específicamente a esa temática identificando dos aspectos principales: los gobiernos urbanos como actores y organizadores de la edificación pública y de la planificación urbanística; y los gobiernos urbanos como reguladores de la construcción privada, pero también como promotores de la seguridad y salud públicas. Se realiza un examen de esos dos papeles fundamentales de las autoridades urbanas y se hacen numerosas referencias a las fuentes documentales sobre las que se basa la investigación. Pero también se ofrece una imagen de la historiografía hasta ahora existente sobre la planificación urbana y las políticas constructivas en las ciudades italianas.

Palabras clave: Planificación urbana; políticas constructivas; construcción pública; construcción privada; estatutos urbanos italianos; petrificación.

ABSTRACT: The essay analyzes the Italian cities' urban planning and building policies, through the study of statutory sources of the twelfth and thirteenth centuries. The text, in particular, faces these matters identifying two main topics: city governments as actors and organizers of public building and urban planning; city governments as regulators of private building, but also as promoters of public safety and health. Trying to examine these two fundamental roles played by city authorities, making many references to the documentary sources on which the research is based, the essay also provides an important picture of the historiography that, up to now, has analyzed the Italian cities' urban planning and building policies.

Keywords: Urban planning; building policies; public building; private building; Italian cities statutes; petrification. 
SUMARIO: 0 Premessa: la ricerca sugli statuti cittadini in Petrifying Wealth. 1 La città come promotore di edilizia pubblica e pianificazione urbanistica, 1.1 La grande edilizia pubblica: palazzi, mura, acquedotti. 1.2 La grande pianificazione urbanistica: nuove lottizzazioni, reti viarie e produttive, interventi programmatici. 2 La città come regolatore: l'edilizia privata e la normativa urbanistica sul benessere collettivo. 2.1 Regolamentazioni per l'edilizia privata. 2.2 Regolamentazioni su igiene e sicurezza. 3 Osservazioni conclusive. 4 Bibliografia.

\section{Premessa: la Ricerca sugli statuti cittadini in Petrifying Wealth}

Nell'ambito del progetto ERC Petrifying Wealth è stata svolta una ricerca sulle politiche urbanistiche ed edilizie delle città italiane, attraverso lo studio dei loro statuti, ovvero le redazioni scritte del complesso delle norme, delle consuetudini e dei provvedimenti che regolavano l'attività degli organismi politici e amministrativi comunali. Il corpus della documentazione esaminata abbraccia sistematicamente tutti gli statuti editi dei centri urbani peninsulari centrosettentrionali, con particolare riferimento a quelli in cui siano riscontrabili regolamentazioni sui temi in questione, all'interno di una cronologia che copre i secoli XII e XIII, sino al 1280, con qualche rarissimo sforamento. Un arco temporale che, quindi, consente di esaminare in maniera puntuale in che modo le politiche delle autorità cittadine si evolsero nel senso di una crescente intenzione e di una crescente capacità di intervento nel settore edilizio e urbanistico.

Le città per le quali si hanno a disposizione tali fonti vanno dall'estremo Nord della Penisola fino ai territori umbri e laziali. L'elenco dei centri che si sono potuti analizzare comprende Aosta, Bassano del Grappa, Bergamo, Biella, Bologna, Como, Genova, Lodi, Milano, Novara, Parma, Pavia, Perugia, Piacenza, Pisa, Pistoia, Reggio Emilia, Sarzana, Savona, Siena, Todi, Treviso, Venezia, Vercelli, Verona, Vicenza, Viterbo e Volterra. L'unica eccezione riguarda un centro attualmente posto immediatamente dopo il confine tra Italia e Francia, ovvero Nizza, a quei tempi però molto vicino alle dinamiche politiche della autonomie cittadine peninsulari. Per alcune di queste città, inoltre, le statuizioni disponibili all'interno dell'arco cronologico considerato sono più d'una, come a Bologna, Genova, Piacenza, Pisa, Pistoia, Reggio Emilia, Sarzana, Siena, Treviso e Viterbo.

Questa indagine ha prodotto un ricco database, in cui sono state schedate tutte le rubriche statutarie utili, indicando per ciascuna la città di riferimento, la datazione, l'edizione di provenienza, l'azione dispositiva, il testo latino con una sua traduzione, le eventuali annotazioni rilevanti e le parole chiave. La distribuzione cronologica delle normative statutarie, all'interno del corpus documentario, è decisamente sproporzionata in favore del Duecento. La quantità di statuti provenienti dal secolo XII, infatti, è esigua e abbraccia solo cinque città: Genova, Piacenza, Pisa, Pistoia e Sarzana ${ }^{1}$.

1 Queste le edizioni di riferimento: Imperiale, Cesare. Codice diplomatico della Repubblica di Genova. Roma: Tipografia del Senato, 1936, pp. 159-165, si tratta della statuizione dei consoli genovesi del 1143; Niccolai, Franco. Contributo allo studio dei più antichi brevi della Compagna genovese. Milano: Giuffrè, 1939, si tratta del breve della Compagna genovese del 1157; Solmi, Arrigo. «Le leggi più antiche del comune di Piacenza». Archivio Storico Italiano, 1915, vol. 75, pp. 65-80, si tratta delle due statuizioni piacentine del 
Lattività dei regimi cittadini quali attori e committenti in campo edilizio, quali promotori di politiche di pietrificazione, spesso monumentali, e di pianificazioni urbanistiche, rappresenta un argomento già ampiamente trattato dalla storiografia degli ultimi cinquant'anni. Non in maniera così sistematica, però, per quanto concerne le normative provenienti dagli statuti. Gli studi più importanti, fino ad ora, si sono concentrati maggiormente su altre categorie di fonti, come quelle notarili - ad esempio contratti di varia natura, vendite, concessioni di terreni edificabili —, quelle prodotte dalle attività assembleari cittadine - come le delibere dei consigli — e quelle materiali, per le analisi di tipo più prettamente archeologico e architettonico. Gli snodi cruciali messi in evidenza da questi studi appaiono principalmente due: la pace di Costanza con l'imperatore Federico I (1183), che consentì alle città dell'area centrosettentrionale italiana di garantirsi, ora anche ufficialmente, quello spazio rilevante di autonomia politica che, di fatto, già si erano costruite, ovvero l'esercizio degli iura regalia in campo amministrativo e giudiziario che le stesse reclamavano da tempo; la crescita del ruolo politico di artigiani e ceti professionali, membri dei gruppi popolari, particolarmente evidente lungo tutto il Duecento ${ }^{2}$.

1170-1171 e del 1181-1182; Banti, Ottavio. I brevi dei consoli del comune di Pisa degli anni 1162 e 1164. Roma: Tipografia del Senato, 1997, pp. 52-100; Vignoli, Paola. I costituti della legge e dell'uso di Pisa (sec. XII). Roma: Tipografia del Senato, 1997, pp. 147-288, si tratta in particolare della statuizione dell'uso pisano del 1186; Berlan, Francesco. Statuti di Pistoia del secolo XII. Bologna: Romagnoli, 1882, volume che comprende tutte le statuizioni pistoiesi, a partire dal 1107 sino ai brevi dei consoli del 1140-1180 e agli statuti del podestà del 1162-1180, parti delle quali (i brevi dei consoli e gli statuti del podestà) sono stati poi riediti più recentemente (ma l'edizione del 1882 resta comunque la più completa); Conti, Mario Niccolò. Corpus statutorum lunigianensium. La Spezia: Accademia Lunigianese di Scienze, 1979, vol. I, pp. 11-16, si tratta delle due statuizioni sarzanesi del 1140 e del 1170 .

2 Per un quadro di riferimento su tali snodi cronologici, a proposito della crescita della capacità politica cittadina in campo edilizio e urbanistico, si rimanda soprattutto a: Maire Vigueur, Jean-Claude. "L'essor urbain dans l'Italie médiévale: aspects et modalités de la croissance». In Europa en los umbrales de la crisis (1250-1350). XXI Semana de estudios medievales de Estella. Pamplona: Gobierno de Navarra, 1996, pp. 171-204; Hubert, Étienne. «La construction de la ville. Sur l'urbanisation dans l'Italie médiévale». Annales. Histoire, Sciences Sociales, 2004/1 (59e année), pp. 109-139. Sull'importanza della pace di Costanza per la storia dell'urbanistica si veda Soldi Rondinini, Gigliola. «Evoluzione politico-sociale e forme urbanistiche nella Padania dei secoli XII-XıII: i palazzi pubblici». In La pace di Costanza 1183. Un difficile equilibrio di poteri fra società italiana ed impero. Convegno Internazionale. Bologna: Cappelli, 1984, pp. 85-98. Sul ruolo svolto dal Popolo nella crescita delle capacità politiche urbanistiche si veda Heers, Jacques. Espaces publics, espaces privés dans la ville. Le Liber terminorum de Bologne 1294. Paris: Editions du centre national de la recherche scientifique, 1984. Per un quadro sulla storia e il ruolo politico-sociale dei regimi popolari cittadini si rimanda a: Menant, François. L'Italia dei comuni (1100-1350). Roma: Viella, 2011, pp. 77-97; Milani, Giuliano. "Contro il comune dei milites. Trent'anni di dibattiti sui regimi di Popolo». In Caciorgna, Maria Teresa, Carocci, Sandro e Zorzi, Andrea (eds.). I comuni di Jean-Claude Maire Vigueur. Percorsi storiografici. Roma: Viella, 2014, pp. 235-258. 


\subsection{La grande edilizia pubblica: palazzi, mura, acquedotti}

Le vicende legate ai palazzi pubblici hanno evidenziato l'importanza del ruolo ricoperto dalla pace di Costanza quale primo snodo chiave per gli interventi edilizi promossi dalle città, anche perché questo evento favorì un consolidamento dell'istituzione del podestà, che aveva affiancato $i$ consoli e andava assumendo un ruolo di primo piano nei governi urbani ${ }^{3}$. Fino al terzo quarto del XII secolo, consoli e podestà si riunivano in locali presi in affitto, spesso dal vescovo o da famiglie del gruppo dirigente, mentre le assemblee si tenevano tra chiese e piazze. Queste pratiche sono testimoniate anche da alcune rubriche statutarie, come a Pistoia, dove nel corso di quello stesso secolo era stata disposta la ricostruzione del palazzo vescovile, opera utile anche per determinate funzioni pubbliche ${ }^{4}$. Dopo la pace di Costanza, invece, cominciarono a comparire i palazzi comunali, il che sanciva, inoltre, la nuova maturità raggiunta dai regimi cittadini ${ }^{5}$. L'erezione di un primo palazzo civico - evento che si verificò praticamente ovunque all'interno dell'area presa in considerazione, in un arco cronologico che si estende sino a circa la metà del Duecento - rispondeva a esigenze sia materiali, sia ideologiche, per un potere ora più stabile e bisognoso di ulteriori spazi per il proprio funzionamento ${ }^{6}$. Questi primi palazzi si collocavano spesso in aree cittadine che simbolicamente richiamavano il passato antico o altomedievale, come a dare continuità e nuova linfa a quel passato ${ }^{7}$. L'affermazione dei governi di Popolo, sostanzialmente nel secondo Duecento, oltre a significare l'avvio di una seconda stagione di costruzione di palazzi civici — poiché le istituzioni si moltiplicavano, diventavano più articolate, e gli edifici esistenti non erano più sufficienti allo svolgimento delle attività di governo e amministrazione - , causò anche una rottura con il passato stesso. Le autorità locali crearono nuovi baricentri urbanistici, spostando il fulcro delle attività civiche in altre aree urbane, anche a simbolo della grande innovazione politica portata dall'evoluzione dei regimi che guidavano le città. A Milano, ad esempio, i governi popolari determinarono l'abbandono del vecchio palazzo comunale (a fianco della cattedrale) in favore di un nuovo edificio, posto all'incrocio dei sei grandi corsi che

3 Il podestà fu un ufficiale inizialmente cittadino, comparso nei governi urbani al tempo di Federico Barbarossa, trasformatosi in forestiero nel corso dell'ultimo quarto del secolo XII. Tale cambiamento portò con sé la professionalizzazione di questa carica e l'avvio di una grande circolazione di podestà tra le diverse città, con conseguente circolazione di competenze e informazioni. Per un quadro si rimanda a: Menant, L'Italia dei comuni, pp. 64-76; Lazzarini, Isabella e Menant, François. «Les podestats». In Caciorgna, Carocci e Zorzi (eds.), I comuni, pp. 177-199. Va precisato che la scansione cronologica riportata nel testo intende riguardare esclusivamente la storia delle vicende legate all'edilizia e all'urbanistica. Per un quadro sulla periodizzazione dell'evoluzione politico-istituzionale dei regimi cittadini, tra seconda metà del secolo xII e prima metà del successivo, si rimanda ai già citati volumi: quello di Menant e quello in onore di Maire Vigueur.

4 Berlan, Statuti di Pistoia, p. 116.

5 Si rimanda soprattutto a Diacciati, Silvia e Tanzini, Lorenzo. «Uno spazio per il potere: palazzi pubblici nell'Italia comunale». In Diacciati, Silvia e Tanzini, Lorenzo (eds.). Società e poteri nell'Italia medievale. Studi degli allievi per Jean-Claude Maire Vigueur. Roma: Viella, 2014, pp. 59-60.

${ }^{6}$ Si veda Maire Vigueur, L'essor urbain, p. 196.

7 Si veda Diacciati e Tanzini, Uno spazio per il potere, pp. 66-67. Un esempio di grande rilievo, inoltre, è la costruzione del nuovo palazzo comunale bolognese, all'inizio del secolo xIII, descritto ampiamente in Bocchi, Francesca. Bologna nei secoli IV-XIV. Mille anni di storia urbanistica di una metropoli medievale. Bologna: Bononia University Press, 2008, pp. 73-78. 
attraversavano i sestieri cittadini, basi della stessa organizzazione popolare ${ }^{8}$. Il grande palazzo pubblico di Orvieto, poi, fu edificato - a partire dal 1280 - non nell'antica platea communis, dove almeno dal 1216 sorgeva il palatium communis presso la chiesa di Sant'Andrea, ma in uno spazio che sarebbe poi divenuto il nuovo centro politico. Così pure a Parma, dove se il palatium vetus occupava l'area della cattedrale, del palazzo vescovile e del battistero, nel corso del Duecento una complessa vicenda costruttiva diede vita a un altro baricentro dell'edilizia pubblica lungo la via Emilia'

Tuttavia va precisato che l'idea della monumentalità, cioè «di un edificio che si staglia in dimensioni ragguardevoli nel paesaggio urbano, con un disegno estetico unitario, leggibile a colpo d'occhio e tale da rappresentare adeguatamente l'identità cittadina nella sua forma più orgogliosa ${ }^{10}$, non si configurava ancora un'esigenza di rilievo nella fase iniziale di costruzione dei nuovi palazzi civici, ma lo sarebbe diventata proprio dalla seconda metà del secolo XIII e, ancor più, durante il secolo successivo; in relazione, cioè, alla comparsa dei regimi popolari prima e di quelli signorili poi ${ }^{11}$. Gli elementi architettonici chiave, tipici soprattutto della prima stagione costruttiva di questi edifici, erano più che altro logge, volte e scale; elementi che, in sintesi, suggerivano come l'esperienza del palazzo pubblico non fosse tanto frontale, quanto quella di uno spazio che si viveva "dal basso» ${ }^{12}$. Non era, allora, la mole complessiva dell'edificio a esprimerne la funzione pratica e simbolica nel tessuto politico urbano, bensì quegli stessi elementi di cui sopra, $\mathrm{i}$ quali rappresentavano le strutture centrali di determinati momenti di vita civica.

Anche analizzando gli statuti è possibile rilevare questa crescente attenzione rivolta ai palazzi pubblici da parte delle autorità cittadine, a partire soprattutto dall'avvio del Duecento. A Pavia, nelle normative redatte tra 1192 e 1206, una rubrica stabiliva che si dovessero individuare tutti gli interventi utili da attuare per il palazzo comunale di Porta Palazzo $^{13}$. Nelle statuizioni bolognesi del 1250 altre rubriche stanziavano il pagamento di 100 soldi locali a Giovanni Tonso, che aveva lavorato alle porte del palazzo comunale, e varavano la predisposizione delle sedute nel palazzo vecchio per lo svolgimento delle

8 Si veda Grillo, Paolo. Milano in età comunale (1183-1276). Istituzioni, società, economia. Spoleto: Fondazione Centro Italiano di Studi sull'Alto Medioevo, 2001, pp. 56-60.

9 Si veda Diacciati e Tanzini, Uno spazio per il potere, p. 67.

10 Diacciati e Tanzini, Uno spazio per il potere, pp. 61-62.

11 Si pensi, ad esempio, alle imprese architettoniche fiorentine avviatesi con l'edificazione del palazzo del Bargello, la cui costruzione ebbe inizio proprio nella seconda metà del Duecento e per il quale si rimanda a Yunn, Amee. The Bargello: a new history of the first communal palace of Florence, 1255-1346. New York: New York University, 2009. Si pensi, inoltre, al lungo percorso di realizzazione del palazzo pubblico senese, nel quale la fase di massima intensità monumentale combaciò con il Governo dei Nove: si rimanda, in particolare, a Balestracci, Duccio e Piccinni, Gabriella. Siena nel Trecento. Assetto urbano e strutture edilizie. Firenze: Nuova Grafica Fiorentina, 1977, pp. 103-106. Sulla politica monumentale dei centri urbani si veda pure Maire Vigueur, Jean-Claude. "La politique monumentale des communes et des seigneuries: un essai de comparaison (Italie centrale, XIV siècle)». In Albonico, Simone e Romano, Serena (eds.). Courts and courtly cultures in early modern Italy and Europe. Roma: Viella, 2016, pp. 37-66.

12 Diacciati e Tanzini, Uno spazio per il potere, p. 64. Sulle questioni architettoniche dell'evoluzione dei palazzi pubblici si rimanda anche a Moretti, Italo. «I palazzi pubblici». In La costruzione della città comunale italiana (secoli XII-inizio XIV). Atti del convegno. Pistoia: Centro Italiano di Storia e Arte, 2009, pp. 67-80.

13 Soriga, Renato. "Il memoriale dei consoli del comune di Pavia». Bollettino della Società Pavese di Storia Patria, 1913, vol. 13, p. 115. 
assemblee ${ }^{14}$. A Perugia, nel 1279, podestà e capitano dovevano far vendere case e casalini posti nel luogo in cui si intendeva edificare il nuovo palazzo, ma intanto dovevano far ristrutturare quello in cui proprio il podestà dimorava; inoltre era ricordato il divieto di occupare le scale esterne nel corso delle sedute assembleari, il che sembra confermare il ruolo chiave di questo genere di elementi architettonici nella mentalità del tempo ${ }^{15}$.

Lestensione delle cinte murarie urbane rappresenta un altro settore nel quale le città si espressero quali attori di edilizia pubblica. In questo ambito non furono tanto singoli eventi, pur di ampio respiro generale, a fare da snodi cronologici nel processo costruttivo, quanto la più costante crescita demografica, che caratterizzò i secoli XII e XIII e determinò la necessità di espandere l'abitato cittadino, inglobando all'interno delle mura aree insediative di recente origine, il che comportava dover ampliare le cerchie esistenti o doverne erigere di nuove. Le ricerche hanno mostrato, per Bologna, Brescia, Ferrara, Firenze, Genova, Milano, Perugia, Pisa, Roma, Venezia, Verona e altri centri, che la costruzione di nuovi quartieri sia stata principalmente promossa dagli enti ecclesiastici — soprattutto monasteri benedettini e capitoli cattedrali - attraverso un programma di lottizzazioni di terreni ad essi appartenenti: i lotti erano distribuiti ai nuovi abitanti per mezzo di contratti ad enfiteusi di lunga durata, dietro compenso iniziale elevato, ma con un censo annuale spesso lieve e con l'obbligo, per l'affittuario, della costruzione di una casa ${ }^{16}$. Le stesse ricerche hanno mostrato che il ruolo dei proprietari laici nell'espansione insediativa fu meno evidente, anche se alcuni casi fanno eccezione: a Genova e a Roma, infatti, una parte dell'habitat urbano si organizzò attorno alle grandi famiglie aristocratiche, rispettivamente i cosiddetti alberghi e i complessi immobiliari dei baroni ${ }^{17}$.

14 Frati, Luigi. Statuti di Bologna dall'anno 1245 all'anno 1267. Bologna: Regia Tipografia, 1876, vol. II, pp. 148, 370-371 e 521 .

15 Bartoli Langeli, Attilio e Caprioli, Severino. Statuto del comune di Perugia del 1279. Perugia: Deputazione di Storia Patria per l'Umbria, 1996, pp. 46-47, 193 e 221-222. Sugli interventi per i palazzi pubblici perugini si rimanda anche a: Silvestrelli, Maria Rita. "L'edilizia pubblica del comune di Perugia: dal palatium comunis al palatium novum populi». In Società e istituzioni dell'Italia comunale: l'esempio di Perugia (secoli XII-XIV). Perugia: Deputazione di Storia Patria per l'Umbria, 1988, vol. II, pp. 482-604; Silvestrelli, Maria Rita. "Grandi cantieri e palazzi pubblici. L'esempio di Perugia». In Crouzet-Pavan, Élisabeth (ed.). Pouvoir et édilité: les grands chantier dans l'Italie communale et seigneuriale. Roma: École Française de Rome, 2003, pp. 105-158.

16 I riferimenti ad alcuni di questi studi sono: Andenna, Giancarlo. «Il monastero e l'evoluzione urbanistica di Brescia tra XI e XII secolo». In S. Giulia di Brescia. Archeologia, arte, storia di un monastero regio dai Longobardi al Barbarossa. Atti del Convegno. Brescia: Grafo, 1992, pp. 93-118; Balestracci e Piccinni, Siena nel Trecento; Bocchi, Bologna; Hubert, Étienne. «Propriété ecclésiastique et croissance urbaine: à propos de l'Italie centro-septentrionale, XIIe-début du XIVe siècle». In Gli spazi economici della chiesa nell'occidente mediterraneo (secoli XII-metà XIV). Atti del convegno. Pistoia: Centro Italiano di Storia e d'Arte, 1999, pp. 125155; Hubert, La construction; Hubert, Étienne. «Urbanizzazione, immigrazione e cittadinanza (XII-metà XIV secolo). Alcune considerazioni generali». In La costruzione della città comunale, pp. 131-145; Maire Vigueur, L'essor urbain; Menant, L'Italia dei comuni, pp. 147-150; Sznura, Franek. L'espansione urbana di Firenze nel Dugento. Scandicci: La Nuova Italia, 1975; Varanini, Gian Maria. "L'espansione urbana di Verona in età comunale: dati e problemi». In Rossetti, Gabriella (eds.). Spazio, società, potere nell'Italia dei comuni. Napoli: Liguori, 1986, pp. 1-25.

17 A tal propósito si vedano: Herres, Jacques. «Urbanisme et structure sociale à Gênes au Moyen Âge». In Heers, Jacques. Société et économie à Gênes (XIVe-XVe siècles). Londra: Variorum, 1979, pp. 371-412; Carocci, Sandro. "Baroni in città. Considerazioni sull'insediamento e i diritti urbani della grande nobiltà». In 
Tuttavia, nel processo di estensione delle cerchie murarie, furono le autorità cittadine a rivestire il ruolo di principali promotori ${ }^{18}$. Tale processo conobbe due fasi di accelerazione, nell'arco cronologico considerato. Le città, infatti, intensificarono la costruzione di fortificazioni soprattutto nella seconda metà del XII secolo e, in seguito, in un periodo che va dalla seconda metà del Duecento sino al secondo quarto del Trecento ${ }^{19}$. L'aumento della superficie fortificata fu notevole più o meno ovunque. Solo per la prima fase gli esempi principali riguardano: Genova, che passò da 22 ettari a 55 nel 1155; Pisa, che passò da 30 a 185 tra 1155 e 1162; Firenze, che passò da 25 a 80 tra 1172 e 1175; Verona, dove il murus novus è attestato nel 1157; Milano, dove le mura furono ricostruite dopo la distruzione della città da parte di Federico I di Svevia, nel 1162, raggiungendo i 240 ettari; Bologna, dove la nuova cerchia detta dei Torresotti fu innalzata a partire dal 1176 e occupò tra i 100 e i 120 ettari $^{20}$. Per la seconda fase, invece, gli esempi più rilevanti riguardano la stessa Bologna: qui la costruzione della Circla — la terza cinta muraria - ebbe inizio nel corso del secondo quarto del secolo xIII, protraendosi fino alla prima metà del Trecento, e arrivò a coprire circa 400 ettari $^{21}$. Ad Assisi, poi, l'ulteriore espansione delle mura avvenne tra 1260 e $1316^{22}$. A Firenze e Siena tali nuovi ampliamenti si verificarono tra la fine del secolo xiII e la prima metà del successivo ${ }^{23}$. A Padova, infine, la fortificazione raggiunse i 450 ettari all'inizio del secolo $\mathrm{XIV}^{24}$.

La storiografia, come anticipato, ha già evidenziato la funzione chiave svolta dai regimi cittadini nell'ambito di questo settore edilizio. Basti pensare a quanto emerge nel volume di Francesca Bocchi a proposito della Circla bolognese: vi si illustra il grande sforzo di collaborazione chiesto dalle autorità urbane alle comunità del contado sia dal punto di vista economico, per l'acquisizione delle superfici territoriali necessarie a comprendere tutte le strutture che avrebbero costituito il complesso delle fortificazioni - fossato, cortina in muratura con terrapieno di rinforzo e due strade, una interna e una esterna - , sia dal punto di vista pratico, per l'ingente spiegamento di mezzi necessari agli scavi. Quanto alle risorse monetarie, infatti, le comunità del contado dovettero

Rome aux XIII et XIVe siècles. Cinq études réunies par Étienne Hubert. Roma: École Française de Rome-Viella, 1993, pp. 137-173; Hubert, Étienne. Espace urbain et habitat à Rome du Xe siècle à la fin du XIIIe siècle. Roma: École Française de Rome, 1990.

18 Sull'ampliamento delle cerchie murarie, oltre ad alcuni degli studi citati nella nota 16 - quelli di Bocchi, Balestracci-Piccinni, Sznura e Varanini —, si vedano anche: De Giovanni, Cesarina. «L'ampliamento di Assisi nel 1316». Bollettino della Deputazione di Storia Patria per l'Umbria, 1975, vol. LXXII, n. ${ }^{\circ}$ I, pp. 1-78; Nicolini, Ugolino. «Le mura medievali di Perugia». In Storia e arte in Umbria nell'età comunale. Atti del convegno. Perugia: Universitá degli Studi di Perugia, 1971, pp. 695-769; Settia, Aldo Angelo. «Cerchie murarie e torri private urbane». In La costruzione della città comunale, pp. 45-66.

19 Si veda Hubert, Urbanizzazione, p. 137.

20 Per questi dati si rimanda a: Heers, Jacques. «Les villes d'Italie centrale et l'urbanisme: origines et affirmation d'une politique (environ 1200-1350)». Mélanges de l'École française de Rome, 1989, vol. 101, n. ${ }^{\circ}$ 1, pp. 72-73; Hubert, La construction, pp. 113-114; Maire Vigueur, L'essor urbain, pp. 176-177.

21 Si veda Bocchi, Bologna, p. 87.

22 Si veda De Giovanni, L'ampliamento di Assisi, pp. 3-33.

23 Si vedano: Hubert, La construction, p. 114; Sznura, L'espansione urbana di Firenze; Balestracci e Piccinni, Siena nel Trecento, pp. 17-29.

24 Si veda Hyde, John Kenneth. Padova nelletà di Dante. Storia sociale di una città-stato italiana. Trieste: LINT, 1985, p. 46. 
essere tassate, ciascuna in relazione al proprio quartiere di riferimento (la suddivisione del contado in quartieri era stata varata nel 1223), per raccogliere così il denaro utile; quanto alle risorse materiali, invece, gli abitanti delle aree extraurbane dovettero fornire in gran numero alla città animali, carri e uomini ${ }^{25}$. Si pensi, inoltre, anche a quanto emerge dal volume di Francesco Pirani su Fermo: in esso, a proposito del programma di espansione urbanistica e di fortificazione avviato dalle autorità pubbliche nel $1241 \mathrm{e}$ realizzato nel giro di una dozzina di anni, è citata la designazione di una commissione che ebbe il compito di espropriare e indennizzare i proprietari dei lotti su cui avrebbe dovuto innalzarsi la cortina muraria ${ }^{26}$. L'iniziativa politica cittadina è altrettanto evidente nel caso dell'ampliamento abitativo e murario di Assisi del 1316, per il quale Cesarina De Giovanni ha messo in luce la rilevanza dello statuto scaturito da tale iniziativa e della magistratura speciale dei Quindici, che doveva occuparsi di organizzare e regolamentare questa grande impresa edilizia, oltre che di acquistare o sgomberare le superfici areali sulle quali si sarebbe poi dovuto edificare ${ }^{27}$.

Le normative statutarie appartenenti al corpus esaminato forniscono dati interessanti sulle iniziative di estensione delle fortificazioni urbane promosse dai regimi politici delle città. A Treviso, nel 1207, il podestà doveva far costruire 50 passi di mura intorno all'abitato, alte e larghe quanto il muro presso la porta dei Santi Quaranta ${ }^{28}$. A Volterra, tra 1210 e 1224, si stabiliva l'ampliamento delle mura cittadine usque ad numerum .C. brachiorum per longitudinem, et per altitudinem usque ad.VI. brachia ${ }^{29}$. A Vercelli, nel 1241, nell'ambito della costruzione della nuova cinta muraria, si fissavano i limiti di spesa in tal senso per il podestà ${ }^{30}$. A Bologna, nelle corpose normative del periodo tra 1245 e 1267, si incontrano diverse rubriche che riguardano interventi sulla $\mathrm{Circla}^{31}$. Anche a Siena, nel 1262, diverse norme programmavano la costruzione della nuova cerchia, con percorso a partire dalla porta Peruzzini ${ }^{32}$. Si interveniva anche su parti del percorso murario che minacciassero di cadere, come a Viterbo nel 1251-1252, oppure per manutenzioni, come a Perugia nel 1279, o ancora per ristrutturare determinati tratti, come a Pisa nel $1286^{33}$.

25 Si veda Bocchi, Bologna, pp. 88-89. Sulla relazione tra l'espansione muraria e la crescita del fisco si veda Menzinger, Sara. "Mura e identità civica in Italia e in Francia meridionale (secc. XII-XIV)». In Menzinger, Sara. Cittadinanze medievali: dinamiche di appartenenza a un corpo comunitario. Roma: Viella, 2017 , pp. 65-111.

26 Si veda Pirani, Francesco. Fermo. Spoleto: Fondazione Centro Italiano di Studi sull'Alto Medioevo, 2010, pp. 134-135.

${ }_{27}$ Si veda De Giovanni, L'ampliamento di Assisi, pp. 7-10.

28 Liberali, Giuseppe. Gli statuti del comune di Treviso. Venezia: Regia Deputazione di Storia Patria per le Venezie, 1950, p. 44.

29 Fiumi, Enrico. Statuti di Volterra, I, (1210-1224). Firenze: Deputazione di Storia Patria per la Toscana, 1951, pp. 34 e 224.

30 Adriani, Giovan Battista. Statuti del comune di Vercelli dell'anno 1241. Torino: Paravia e comp., 1877, pp. 188-189.

31 Frati, Statuti di Bologna, pp. 340-514.

32 Zdekauer, Lodovico. Il costituto del comune di Siena dell'anno 1262. Milano: Hoepli, 1897, pp. 279-284.

33 Federici, Vincenzo. Statuti della provincia romana. Roma: Tipografia del Senato, 1930, vol. II, p. 163; Bartoli Langeli e Caprioli, Statuto, pp. 66-72; Bonaini, Francesco. Statuti inediti della città di Pisa dal XII al XIV secolo, I. Firenze: Vieusseux, 1854, p. 477. 
Del resto la presenza in alcune città di ufficiali come i magistri murorum, attestati ad esempio proprio a Pisa già nel breve dei consoli del $1162^{34}$, certifica ulteriormente come le autorità politiche locali si occupassero in maniera diretta delle cinte murarie fin dalla seconda metà del secolo XII.

In tema di fonti per l'approvvigionamento idrico, le ragioni dalle quali scaturì l'avvio di determinate imprese architettoniche non furono soltanto legate alla già richiamata evoluzione politica dei centri urbani, ma anche a questioni di natura geografica. Fu senz'altro la crescente capillarità di intervento dei regimi cittadini in ogni ambito della vita civica a consentire la programmazione di certe costruzioni; tuttavia il problema di come fornire l'acqua alla cittadinanza crebbe di pari passo con la costante espansione demografica, riguardando in particolar modo le città appartenenti a una fascia più centrale dell'area considerata, ovvero quelle realtà poste nel contesto delle zone montane e collinari tra le attuali Toscana, Umbria, Marche e Lazio. Città quasi sempre situate su alture, in cui pertanto era necessario ingegnarsi più che altrove per far sì che le acque fossero fruibili a tutti. La storiografia ha messo in rilievo alcune di queste imprese architettoniche, soprattutto nei casi di Orvieto, Perugia e Siena, dove gli interventi più significativi si concentrarono nella seconda metà del secolo $\mathrm{XIII}^{35}$ : si tratta dell'acquedotto dell'Alfina orvietano ${ }^{36}$, della Fontana Maggiore e dell'acquedotto di Montepacciano perugini $^{37}$ e della Fonte Branda senese, con relativo acquedotto ${ }^{38}$. È evidente che il clima politico nel quale queste imprese architettoniche videro la luce risultò decisivo per la loro messa in opera, come mostra il caso perugino: il decollo politico-economico della città, nel corso della prima metà del Duecento, portò il regime popolare, dagli anni Settanta dello stesso secolo, a trovare «i mezzi economici, le capacità organizzative, le motivazioni ideologiche per esprimere e materializzare i sogni di una comunità che vuole essere grande» ${ }^{39}$.

L'analisi degli statuti consente di far emergere, ancor più chiaramente, il ruolo di grande attore svolto dall'autorità cittadina negli interventi edilizi per l'approvvigionamento delle acque, soprattutto a partire dal secondo quarto del secolo XIII. Rubriche statutarie relative all'edificazione di nuovi acquedotti, anche molto dettagliate, si incontrano a Verona (1228), a Viterbo (1251-1252) e a Perugia $(1279)^{40}$. Nelle normative bolognesi del 1250, inoltre, due capitoli stabilivano la ristrutturazione di pozzi presenti

34 Banti, I brevi, p. 60.

35 Si veda Maire Vigueur, L'essor urbain, p. 201.

36 Si rimanda a Riccetti, Lucio. La città costruita. Lavori pubblici e immagine in Orvieto medievale. Firenze: Le Lettere, 1992, pp. 293-333.

37 Si rimanda a Silvestrelli, Grandi cantieri, pp. 110-113, ma anche a Silvestrelli, L'edilizia pubblica, articolo nel quale è indicata altra bibliografia utile sull'acquedotto di Montepacciano e la Fontana Maggiore.

38 Si rimanda a Bargagli Petrucci, Fabio. Le fonti di Siena e i loro acquedotti. Siena-Firenze-Roma: Olschki, 1906, ma anche a Balestracci e Piccinni, Siena nel Trecento, pp. 146-147.

39 Silvestrelli, Grandi cantieri, p. 110.

40 Liber iuris civilis urbis Veronae, per Bartholomaeum Campagnolam. Verona: apud Petrum Antonium Bernum, 1728, p. 109; Federici, Statuti, pp. 113-117; Bartoli Langeli e Caprioli, Statuto, pp. 177-178, proprio a proposito dei lavori per l'acquedotto di Montepacciano. Per quanto riguarda il caso viterbese di metà Duecento, inoltre, si veda Balestracci, Duccio. «La politica delle acque urbane nell'Italia comunale». Mélanges de l'École Française de Rome, 1992, vol. 104, p. 463, dove si citano gli statuti in relazione alla progettazione 
in varie contrade, mentre una programmava la derivazione de aqua savine in tutte le contrade urbane ${ }^{41}$. Nel costituto senese del 1262 una rubrica stabiliva la riparazione di pozzi e cloache presenti entro le mura cittadine, un'altra ordinava la sistemazione del flusso d'acqua che correva per via di Galgaria ${ }^{42}$. A Todi, nel 1275, un capitolo incaricava il podestà, con i maestri delle pietre e i muratori, di fare in modo che tutte le acque partissero e giungessero alla fonte Scarnabicci, mentre un altro lo incaricava di far ristrutturare da due buoni maestri l'acquedotto attraverso il quale le acque fluivano verso le cisterne della piazza comunale ${ }^{43}$. Infine gli statuti perugini del 1279 contano ben cinque rubriche in cui si disponevano riparazioni e sistemazioni di fontane, mentre una stabiliva la nomina di un magister per ogni porta della città che, nella propria area di riferimento, procedesse a far riparare anche le fognature ${ }^{44}$.

\subsection{La grande pianificazione urbanistica: nuove lottizzazioni, reti viarie e produttive, interventi programmatici}

I centri urbani furono altrettanto attivi come promotori di innovativi piani urbanistici, anche in questo caso specialmente a partire dal secondo quarto del Duecento. La «trasformazione dello spazio urbano è forse la maggiore caratteristica dell'ascesa al potere dei gruppi popolari» ${ }^{45}$; i nuovi regimi politici, pertanto, oltre ad aver bisogno di più spazi ed edifici per lo svolgimento delle funzioni civiche, intendevano creare contesti urbani più a misura di una cittadinanza ulteriormente cresciuta e stratificata. Le preoccupazioni in tema di approvvigionamento delle acque, come pure gli interventi di estensione delle cerchie murarie, hanno in parte già mostrato tali intenti. Fanno ancora più luce le politiche portate avanti in altri settori, a partire da quello dell'estensione dell'abitato.

Non c'è dubbio che gli enti ecclesiastici — come già ricordato — abbiano inizialmente rivestito un ruolo primario nell'ampliamento delle aree insediative urbane. Anzi, molta storiografia ha ritenuto eccezionali i casi di Genova e Venezia, dove le strategie urbanistiche operate dai poteri pubblici furono notevoli e più precoci: a Genova, già a partire dagli anni Trenta del secolo XII, si fecero edificare nuove strutture e si regolamentò l'esistente, disciplinando la larghezza di alcune vie e l'altezza delle torri, vietando i ponti di legno colleganti case, accertando gli spazi dedicati ai mercati, intervenendo sul Molo, sull'organizzazione della Ripa, ovvero il porticato pubblico lungo un chilometro, con colonne di pietra, addossato a edifici privati, che regolava l'accesso ai quartieri retrostanti il porto grazie a una serie di vie tangenziali ${ }^{46}$; a Venezia, già dalla prima parte

dell'acquedotto sotterraneo coperto planellis, al quale avrebbero potuto attingere i privati le cui case si trovassero lungo il suo corso, e a spese dei quali l'intervento veniva finanziato.

${ }_{41}$ Frati, Statuti di Bologna, pp. 361, 401-402 e 416.

42 Zdekauer, Il costituto, pp. 277-278 e 288.

43 Ceci, Getulio e Pensi, Giulio. Statuto di Todi del 1275. Todi: Trombetti, 1897, pp. 91-92 e 104105.

44 Bartoli Langeli e Caprioli, Statuto, pp. 172-179 e 197.

45 Riccetti, La città costruita, p. 111.

46 Si veda Guglielmotti, Paola. Genova. Spoleto: Fondazione Centro Italiano di Studi sull'Alto Medioevo, 2013, p. 14. 
del Duecento, il Gran consiglio sostituì i privati nell'iniziativa, individuando gli spazi da prosciugare per potervi costruire; più avanti fu istituita una magistratura specifica - i giudici del piovego - che doveva supervisionare l'urbanizzazione e farne rispettare le regole ${ }^{47}$. Tuttavia, dal secondo quarto del secolo xIII, le normative statutarie mostrano come le autorità cittadine iniziarono a prendere maggiore iniziativa nella progettazione di nuove aree insediative e nella programmazione dell'immigrazione. A Volterra, nel 1224, si regolamentava la vendita di spazi per la costruzione di nuove case, prescrivendo dettagli e particolarità degli edifici da realizzare, come in una lottizzazione ${ }^{48}$. Anche a Treviso, tra 1231 e 1260, si stabilivano norme edilizie per la realizzazione di nuove case, in città e presso una terra di nuova fondazione come Castelfranco; da notare l'obbligo di costruire abitazioni di rilievo maggiore per chi possedesse un patrimonio stimato di più di 1500 lire e, proprio a Castelfranco, il divieto di innalzarle altior octo punctis ${ }^{49}$. A Viterbo, nel 1251-1252, si disponeva che chiunque volesse acquisire la cittadinanza dovesse acquistare una casa, o un casalino su cui realizzarl ${ }^{50}$. A Siena, nel 1262, veniva vietata la costruzione di abitazioni nei sobborghi al di fuori degli spazi assegnati, per dieci anni, e si programmava l'edificazione di un nuovo borgo in Paganico ${ }^{51}$.

Altro settore in cui le città promossero una vera e propria pianificazione fu quello delle reti viarie. Tra le ragioni principali individuate da Thomas Szabó risultano particolarmente rilevanti la stessa estensione dell'abitato, che determinava la necessità di aprire nuovi percorsi stradali, e la creazione di condizioni di vita migliori per la collettività ${ }^{2}$. Queste politiche di pianificazione viaria si incontrano già nel corso del secolo XII, come ad esempio a Pisa e Pistoia: nel breve dei consoli pisani del 1162 si parlava di «viis iuxta muros noviter factis» e di espropriazioni avvenute a questo scopo; nel breve dei consoli pistoiesi del 1140-1180 si disponeva che le nuove strade, intorno a tutta la città, dovessero essere larghe 12 piedi $^{53}$. Durante il Duecento, poi, si diffuse notevolmente l'uso di pavimentare le strade mediante lastricatura e selciatura. Gli esempi principali, dall'esame della storiografia, sono quelli di Bologna, Siena e Vicenza ${ }^{54}$. L'analisi delle normative statutarie consente di ampliare lo sguardo: i centri urbani più attivi negli interventi sulla rete viaria, infatti, appaiono proprio Bologna (1250), ma anche Reggio Emilia (1242) e

47 Si vedano Crouzet-Pavan, Élisabeth. «Sopra le acque salse»: espaces urbains, pouvoir et société à Venise à la fin du Moyen Âge. Paris e Roma: École Française de Rome, 1992 e Orlando, Ermanno. Venezia. Spoleto: Fondazione Centro Italiano di Studi sull'Alto Medioevo, 2016, pp. 9-10.

48 Fiumi, Statuti di Volterra, pp. 180-181.

49 Liberali, Gli statuti, pp. 196, 253 e 288.

50 Federici, Statuti, p. 179.

51 Zdekauer, Il costituto, pp. 286 e 383.

52 Si rimanda a Szabó, Thomas. "Genesi e sviluppo della viabilità urbana». In La costruzione della città comunale, pp. 149-162, ma anche a Szabó, Thomas. «Il controllo dello spazio e la genesi della rete viaria comunale nel Medioevo». In Rossetti (ed.), Spazio, società, potere, pp. 27-36.

53 Banti, I brevi, p. 65 e Berlan, Statuti di Pistoia, pp. 88-90.

54 Si rimanda a: Bocchi, Bologna, pp. 98-99; Balestracci e Piccinni, Siena nel Trecento, pp. 41-44, dove si parla degli interventi anche duecenteschi; Lomastro, Francesca. Spazio urbano e potere politico a Vicenza nel XIII secolo. Vicenza: Accademia Olimpica, 1981, pp. 53-60. 
Perugia $(1279)^{55}$. Inoltre a Novara, nel 1277, una rubrica stabiliva che le strade cittadine andassero tutte pavimentate, alla stessa maniera di quelle che già lo fossero ${ }^{56}$. In vari casi, poi, esistevano ufficiali addetti alla custodia delle vie, come mostra ad esempio il caso viterbese nella statuizione del 1251-125257. Questo genere di interventi interessò anche la rete dei ponti. Nelle fonti statutarie studiate, i capitoli in cui se ne disponeva la costruzione e/o la selciatura/lastricatura sono molto ricorrenti: le città più operose in tal senso appaiono Bologna (1250), Treviso (1231-1260), Parma (1255), Siena (1262), Novara (1277), Perugia (1279), Pisa (1286) ${ }^{58}$. I materiali utilizzati per l'edificazione erano prevalentemente legno e pietra, a seconda delle dimensioni e del peso da sostenere. Ancora a proposito di pavimentazione, va aggiunto come la ricerca svolta abbia evidenziato una forte congruenza tra le norme degli statuti di Bologna e Reggio Emilia, soprattutto in certa terminologia: «salegare» o "sallegare» sono verbi che si incontrano solo in queste statuizioni per indicare l'operazione di selciatura/lastricatura. La circolazione di modelli documentari, pertanto, in questo caso appare chiara proprio in area emiliana.

La pianificazione urbanistica attuata dai regimi cittadini interessò anche l'edilizia legata al settore della produzione artigianale e manifatturiera. Il tema della distribuzione delle attività produttive nel tessuto cittadino è stato già affrontato dalla storiografia, ma in maniera approfondita solo per pochi casi ${ }^{59}$. L'esempio principale è Genova, dove alcuni toponimi - come campetus fabrorum, carrubeus ferrariorum e altri - segnalavano le aree in cui si collocavano le varie produzioni ${ }^{60}$. Entrando più nello specifico, grazie all'ausilio delle fonti statutarie si riscontrano diversi interventi di costruzione di nuovi mulini nel territorio ${ }^{61}$, non solo da affidare in conduzione ma anche direttamente gestiti dai comuni ${ }^{62}$; in certi casi veniva regolamentata la struttura di questi fabbricati, come

55 Citando soltanto l'edizione mai menzionata sin qui, le rubriche dedicate alle strade sono almeno una sessantina nella statuizione bolognese del 1250, meno di una decina in quella reggiana del 1265 (Cerlini, Aldo. Consuetudini e statuti reggiani del secolo XIII. Milano: Hoepli, 1933) e una decina in quella perugina del 1279. La sproporzione in favore di Bologna dipende anche dalla vastità delle sue statuizioni duecentesche rispetto a qualunque altro caso.

56 Ceruti, Antonio. Statuta communitatis Novariae anno 1277. Novara: Fratelli Miglio, 1878, pp. 76-77.

57 Federici, Statuti, pp. 113-117.

58 Citando soltanto l'edizione mai menzionata sin qui, le rubriche dedicate ai ponti sono almeno una trentina negli statuti di Bologna del 1250, una decina in quelli di Treviso del 1231-1260, una ventina in quelli di Parma del 1255 (Ronchini, Amadio. Statuta communis Parmae digesta anno 1255. Parma: Officina Fiaccadori, 1856), una ventina in quelli di Siena del 1262, cinque in quelli di Novara del 1277, un'altra ventina in quelli di Perugia del 1279 e una decina in quelli di Pisa del 1286.

59 Si veda Franceschi, Franco. "I paesaggi della produzione». In La costruzione della città comunale, p. 173.

60 Si rimanda a Grossi Bianchi, Luciano e Poleggi, Ennio. Una città portuale nel medioevo: Genova nei secoli X-XVI. Genova: Sagep, 1980, pp. 32, 71, 75-76.

${ }_{61}$ Come a Milano (1216), Volterra (1210-1222), Siena (1262) e Reggio Emilia (1265): Besta, Enrico e Barni, Gian Luigi. Liber consuetudinum Mediolani anni MCCXVI. Milano: Giuffrè, 1949, p. 107; Fiumi, Statuti di Volterra, pp. 351-354; Zdekauer, Il costituto, p. 43; Cerlini, Consuetudini, pp. 177-178. Alcuni mulini erano anche posseduti in consorzio, come mostra una rubrica di Todi del 1275: Ceci e Pensi, Statuto di Todi, p. 83.

${ }_{62}$ Sulla questione si rimanda a Bortolami, Sante. "Acque, mulini e folloni nella formazione del paesaggio urbano medievale (secoli XI-XIV)». In Paesaggi urbani dell'Italia padana nei secoli VIII-XIV. Bologna: Cappelli, 1988, pp. 277-330. 
soprattutto a Bologna nel $1250^{63}$. Sulle fucine è particolarmente rilevante una norma proveniente da Bassano Del Grappa (1259), che indicava ai fabbri di adattare le loro fucine «cum muro et alio aptamento eis oportuno, quod occasione ignis ipsarum fuscinarum non possit aliquod dampnum inde venire» ${ }^{64}$. Sulle fornaci, poi, si nota soprattutto l'intento di farne costruire in alcuni borghi o castelli del territorio: così a Vercelli, nel 1241, e a Bologna, nel $1250^{65}$. A Treviso, tra 1231 e 1260, si davano anche precisi riferimenti al loro posizionamento, come in una sorta di organizzazione pubblica di zone produttive ${ }^{66}$. A tali politiche si affiancavano quelle sui materiali edilizi. Spesso le autorità cittadine fissavano i prezzi di pietre, mattoni, calce, tegole, coppi, quadrelli e altro ancora, come pure i modelli di tali elementi, per fornire un indirizzo architettonico di urbanizzazione ${ }^{67}$. Anche in quest'ultimo caso il formulario delle norme mostra forti somiglianze: spesso, pur se in realtà diverse, si incontrano rubriche statutarie in cui si disponeva che mattoni e/o coppi dovessero essere prodotti «ad modum [modulum/mogellum] comunis». Infine a Treviso, nel 1218, si concedeva alle fornaci maggiori di cuocere oltre i 12.000 mattoni e a quelle minori di cuocerne oltre i 7.000; tra 1231 e 1260, invece, si disponeva che mattoni e pietre dovessero essere prodotti boni, pleni et perfecti, senza alcun cavamento ${ }^{68}$.

Alle fasi di ascesa e maturazione dei regimi popolari, o comunque della forte influenza popolare sulla vita politica cittadina — più o meno lungo tutto il Duecento corrisposero anche una serie di interventi, fortemente programmatici, di ulteriore trasformazione dello spazio urbano. Si fa riferimento, in particolare, all'apertura di nuove piazze - con conseguenti adeguamenti dell'edilizia per i mercati, degli accessi a tali spazi, dei porticati, eccetera - affinché esse divenissero i nuovi fulcri civici e commerciali delle città ${ }^{69}$. I casi principali su cui la storiografia ha già fatto luce sono soprattutto Bologna (i lavori per la Piazza Maggiore, a partire dall'inizio del secolo XIII ${ }^{70}$ ), Milano (l'organizzazione degli spazi aperti adiacenti al Broletto Nuovo, a partire dal $1228^{71}$ ) e Parma

63 Frati, Statuti di Bologna, pp. 207-210. Norme meno dettagliate, ma di stessa natura, anche a Treviso, tra 1231 e 1260 (Liberali, Gli statuti, p. 242), e Todi, nel 1275 (Ceci e Pensi, Statuto di Todi, p. 37).

64 Fasoli, Gina. Statuti del comune di Bassano dell'anno 1259 e dell'anno 1295. Venezia: Regia Deputazione di Storia Patria per le Venezie, 1940, p. 133.

65 Adriani, Statuti, p. 174; Frati, Statuti di Bologna, pp. 357, 396 e 411-412.

66 Liberali, Gli statuti, p. 272.

67 Citando soltanto le edizioni mai menzionate sin qui, ciò accadeva a Bergamo (1237: "Antiquae collationes statuti veteris civitatis Pergami». In Historiae patriae monumenta edita iussu Regis Karoli Alberti, tomus XVI, Leges municipales, tomus secundus, pars altera. Aosta: Fratelli Bocca, 1876, pp. 2009-2010), Biella (1245: Gabotto, Ferdinando. "Gli statuti di Biella secondo il codice originale del 1245». Biblioteca della società storica subalpina, 1908, vol. 34, pp. 363-364), Bologna (1250), Novara (1277), Parma (1255), Reggio Emilia (1265-1268), Savona (primo quarto del secolo XIII: Calleri, Marta. «I più antichi statuti di Savona». Atti della società ligure di storia patria, 1997, nuova serie, vol. 37, n. ${ }^{\circ}$ II, pp. 181-182), Siena (1250: Banchi, Luciano. "Breve degli officiali del comune di Siena compilato nell'anno 1250». Archivio Storico Italiano, 1866, serie terza, tomo III, parte II, pp. 99-100; ma anche 1262), Viterbo (1251-1252) e Vercelli (1241).

68 Liberali, Gli statuti, pp. 106 e 152-153.

69 Si veda Crouzet-Pavan, Élisabeth. «La cité communale en quête d'elle-même: la fabrique des grands espaces publics». In La costruzione della città comunale, pp. 111-118.

70 Si rimanda a Bocchi, Bologna, pp. 73-78.

71 Si rimanda a Grillo, Milano, pp. 56 e seguenti. 
(la realizzazione della platea nova, tra 1221 e $1224^{72}$ ). Dall'analisi degli statuti emerge che per alcune città di mare, come ad esempio Pisa, preoccupazioni simili da parte delle autorità locali si iniziano a incontrare già nella seconda metà del secolo XII: nel 1164, infatti, una rubrica del breve dei consoli pisani disponeva che costoro si impegnassero a chiedere consiglio ai senatori su una serie di interventi relativi a tende e banchi dei mercanti posti in pubblico ${ }^{73}$. Tuttavia fu nel Duecento che tale interesse si moltiplicò. A Treviso, tra 1231 e 1260, alcune norme stabilivano lo svuotamento della piazza di San Leonardo da qualunque edificio, mediante anche la distruzione di botteghe e case, se necessario ${ }^{74}$. I piani urbanistici senesi, invece, sono evidenti nel costituto del 1262, in cui più capitoli si occupavano della Piazza del Campo, ordinandone lo sgombero da ogni costruzione, vietando l'edificazione e stabilendo che i portici della piazza fossero consentiti solo se alti 8 braccia e larghi 3; le ragioni vanno individuate soprattutto nel fatto che si trattasse di un'area di mercato ${ }^{75}$.

Questo tipo di programmazione urbanistica, matura e consapevole, toccava dunque ormai tutti i settori. Tanto che, proprio negli statuti della seconda metà del secolo XIII, si incontrano alcuni capitoli che affrontano in maniera complessiva la questione della pianificazione e della trasformazione dello spazio urbano. A Reggio Emilia, ad esempio, nel 1265 una norma incaricava il podestà di nominare un addetto a supervisionare tutte le strutture edilizie spettanti al comune, nonché a individuare quelle da edificare a spese pubbliche, tanto in città quanto nel territorio, come mura, porte, canali, ponti ${ }^{76}$. Ancor più imponenti le tre rubriche pisane, dalla statuizione del 1286, in cui si elencavano $\mathrm{i}$ lavori da fare in diverse aree urbane e comitali, relativamente a ponti, mura, torri, acquedotti, canali, scalinate di discesa all'Arno e scarichi fognari ${ }^{77}$. Una programmazione oramai totale. Quella programmazione che promosse realizzazioni decisamente spettacolari, come a Bologna e Firenze, i cui regimi politici, tra XIII e XIV secolo, rimodellarono completamente le due città ${ }^{78}$.

\section{LA CITTÀ COME REGOLATORE: L'EDILIZIA PRIVATA E LA NORMATIVA URBANISTICA SUL BENESSERE COLLETTIVO}

Gli snodi cronologici cui si è già fatto riferimento rappresentano momenti in buona parte decisivi anche per l'altro grande ruolo ricoperto dalle autorità cittadine nel proces-

72 Si rimanda a Crouzet-Pavan, La cité communale, p. 112, ma anche a Ronchini, Statuta communis Parmae, pp. 85-86.

${ }_{73}$ Banti, I brevi, p. 100.

74 Liberali, Gli statuti, pp. 46 e 277.

75 Zdekauer, Il costituto, pp. 287-291.

76 Cerlini, Consuetudini, pp. 117-119.

77 Bonaini, Statuti inediti, pp. 467-475 e 512-518.

78 Si rimanda a: Bocchi, Bologna, pp. 87-144; Milani, Giuliano. Bologna. Spoleto: Fondazione Centro Italiano di Studi sull'Alto Medioevo, 2012, pp. 1-5 e 147-170; Fanelli, Giovanni. Firenze. Bari: Laterza, 1980; Tanzini, Lorenzo. Firenze. Spoleto: Fondazione Centro Italiano di Studi sull'Alto Medioevo, 2016, pp. 192-207. 
so di urbanizzazione: quello di regolatore. Senza dubbio la maggiore autonomia offerta dalla pace di Costanza, la stabilizzazione dell'ufficio podestarile - a cavallo tra la fine del secolo XII e l'avvio del successivo - e la crescita, soprattutto dalla metà del Duecento, della capacità operativa scaturita dall'ascesa politica dei gruppi popolari, consentì ai regimi urbani di mettere in piedi regolamentazioni sempre più capillari che andarono a disciplinare sia l'edilizia privata, sia l'edilizia legata alla sicurezza dei cives e all'igiene pubblica $^{79}$. Settori, dunque, in cui la città non agiva quale attore, bensì quale normatore. Tuttavia, nel campo del privato, la cronologia degli interventi non rispetta pedissequamente tale periodizzazione. Vediamo, più rapidamente, come e perché.

\subsection{Regolamentazioni per l'edilizia privata}

La storiografia si è occupata principalmente dell'edilizia privata delle grandi famiglie aristocratiche e delle tipologie dominanti delle abitazioni dei cittadini. Gli studi si sono concentrati sulle caratteristiche di torri e case-torri appartenenti ai casati delle élites urbane, da un lato simboli di uno stato sociale elevato, dall'altro strumenti di difesa e offesa nel contesto dei conflitti locali ${ }^{80}$. Per quanto riguarda le case, poi, le analisi hanno contribuito a renderne più chiari diversi elementi, quali forme, dimensioni, materiali utilizzati per la costruzione, sistemazione degli interni, strutture e ambienti di servizio ${ }^{81}$. L'analisi degli statuti, invece, consente di fare luce su come le autorità urbane procedettero nel regolamentare queste attività edilizie, che è quanto maggiormente preme.

Il settore in cui le politiche cittadine si mossero più precocemente appare quello dei conflitti tra famiglie aristocratiche e tra fazioni, che investivano anche gli edifici privati. Le città dalle cui statuizioni proviene il maggior numero di norme sulla guerra di torre sono Genova (1143), Pisa (1162-1164 e 1286), Pistoia (1141-1180), Verona (1228), Vi-

79 Quell'edilizia che consente di parlare di «modernità» delle città italiane tra Duecento e Trecento: si veda Bocchi, Francesca. "La modernizzazione delle città medievali». In La costruzione della città comunale, pp. 333-347.

${ }_{80}$ Si rimanda soprattutto a: Bocchi, Bologna, pp. 45-49; De Minicis, Elisabetta. Case e torri medievali I. Atti del Convegno La città e le case: tessuti urbani, domus e case-torri nell'Italia comunale. Roma: Edizioni Kappa, 1996; De Minicis, Elisabetta e Guidoni, Enrico. Case e torri medievali II. Atti del Convegno La città, le torri e le case: indagine sui centri dell'Italia comunale. Roma: Edizioni Kappa, 2001; De Minicis, Elisabetta e Guidoni, Enrico. Case e torri medievali III, Atti del Convegno Case e torri medievali: indagine sui centri dell'Italia comunale. Roma: Edizioni Kappa, 2005; Settia, Aldo Angelo. «Lo sviluppo di un modello: origine e funzioni delle torri private urbane nell'Italia centrosettentrionale». In Paesaggi urbani dell'Italia padana, pp. 155-171; Varanini, Gian Maria. "Torri e casetorri a Verona in età comunale: assetto urbano e classe dirigente». In Paesaggi urbani dell'Italia padana, pp. 173-249.

81 Oltre ai volumi di De Minicis e Guidoni, elencati nella nota precedente, altre analisi rilevanti sulle abitazioni private sono: Balestracci, Duccio. «Immigrazione e morfologia urbana nella Toscana bassomedievale». In Maire Vigueur, Jean-Claude (ed.). D'une ville à l'autre. Structures matérielles et organisation de l'espace dans les villes européennes (XIIIe - XVIe siècle). Actes du colloque organisé par l'École Française de Rome. Paris: École Française de Rome, 1989, pp. 87-105; Balestracci e Piccinni, Siena nel Trecento, pp. 77-102; Sznura, L'espansione urbana di Firenze, pp. 23-39. 
terbo (1237-1238 e 1251-1252), Volterra (1210-1224) e Treviso (1218 e 1231-1260) ${ }^{82}$. A Genova, Pisa e Pistoia tale precocità è evidente: in questo campo le autorità locali dovettero intervenire già nel corso del secolo XII. La regolamentazione dell'altezza di torri e case-torri, infatti, era necessaria per la gestione di questi conflitti già in una società ancora poco articolata e prevalentemente magnatizia, dal momento che i grandi casati spesso combattevano tra loro proprio da certe altezze, scagliando pietre o dardi contro persone o immobili e, a volte, assaltando edifici altrui. Esemplare la situazione genovese, dove la statuizione del 1143 obbligava coloro che possedevano torri a giurare di farle abbassare per undici anni a una misura concordata, vietando di edificarne più alte di 80 piedi $^{83}$. Le sanzioni, un po' ovunque e anche più avanti nel tempo, prevedevano da risarcimenti pecuniari, in caso di danni alle cose, fino alla distruzione della propria torre, in caso di danni alle persone e omicidi ${ }^{84}$. A Treviso, nel 1218, una norma vietava agli stessi consoli di usare la torre concessagli dal podestà per fare guerra, mentre tra 1231 e 1260 si stabilivano pene pecuniarie per chi non consegnasse alle autorità cittadine torri da esse richieste ${ }^{85}$. A Pisa, nel 1286, in presenza di due o più consortes nella proprietà di torri o case, i quali commettessero maleficium, i loro beni comuni andavano distrutti e dovevano prima essere stimati da un agrimensore e dei magistri ${ }^{86}$. In questo settore, inoltre, al di là della netta somiglianza delle tipologie di interventi dispositivi adottati da varie città - che di per sé è un elemento interessante, ma comunque legato all'ovvia somiglianza materiale delle torri —, appare simile anche il formulario delle norme tra realtà diverse: capita spesso, infatti, di incontrare rubriche statutarie in cui si legge che si quis/si aliquis avrebbe commesso tale reato avrebbe poi scontato quella determinata pena, oppure in cui si legge che le autorità iurare faciant che chi possiede torri faciant ipsas diminuere usque ad una precisa misura.

Altro campo in cui i regimi politici, in maniera cronologicamente crescente, regolamentarono l'edilizia privata furono le modalità di occupazione degli spazi pubblici con costruzioni, per evitare abusi. Una certa precocità, qui, riguarda solo Pisa (1162), dove una rubrica del breve dei consoli vietava l'edificazione di ballatoi permanenti, a scopo commerciale, sulle vie e lungo la riva dell'Arno ${ }^{87}$, e Pistoia (1162-1180), dove un capitolo degli statuti del podestà incaricava quest'ultimo di far distruggere alcuni balconi per i quali erano stati vietati presenza e rifacimento ${ }^{88}$. Disposizioni simili, più avanti nel tempo e relative non soltanto a ballatoi, ma anche a banchi, deschi, a elementi come finestre,

82 In molte altre città del campione documentario studiato compaiono queste norme, in maniera meno ricorrente.

83 Imperiale, Codice diplomatico, pp. 163-165.

84 Lo mostrano soprattutto le norme di Genova, Pisa e Pistoia nel secolo XII, che si ritrovano poi nel Duecento in centri come Verona, Viterbo e Volterra: Imperiale, Codice diplomatico, p. 159; Banti, I brevi, pp. 60-61 e 88; Berlan, Statuti di Pistoia, pp. 75-76, 109-110 e 117; Liber iuris civilis urbis Veronae, pp. 52, 70 e 207-208; Federici, Statuti, pp. 74, 184, 198-199 e 227-228; Fiumi, Statuti di Volterra, pp. 26, 75-76 e 210 211. Sul tema delle demolizioni punitive si rimanda a Mucciarelli, Roberta. «Demolizioni punitive: guasti in città». In La costruzione della città comunale, pp. 293-330.

85 Liberali, Gli statuti, pp. 76, 108 e 116.

86 Bonaini, Statuti inediti, pp. 402-403.

87 Banti, I brevi, p. 62.

88 Berlan, Statuti di Pistoia, pp. 31 e 110-111. 
portici, solaria e scale esterne, per aree cittadine di volta in volta specificate, si riscontrano nelle statuizioni di Como (1210), Volterra (1224), Treviso (1230-1231), Vercelli (1241), Biella (1245), Bologna (1250), Siena (1250 e 1262), Reggio Emilia (1268), Todi (1275), Novara (1277), Perugia (1279) e ancora Pisa $(1286)^{89}$. Inoltre, alcune norme sullo sgombero delle vie per non intralciare la circolazione ci giungono da Vercelli (1241), Siena (1250) e Reggio Emilia (1270) ${ }^{90}$.

Nell'edilizia privata le autorità, in particolare dal secondo quarto del secolo XIII, seppero intervenire anche su dettagli tecnici e per alcune concessioni. Gli esempi sono vari: a Treviso, tra 1231 e 1260, si stabiliva la copertura degli androni ${ }^{91}$ privati currentes verso un portico, tantum quantum porticus protenduntur ${ }^{92}$. Ad Aosta, nel 1253, si disponeva che gli abitanti di una determinata area (quella donata alla città dal conte sabaudo Tommaso) fossero tenuti a dare 12 denari all'anno al conte episcopo per estendere la propria abitazione nella parte anteriore, secondo larghezza, ad eccezione di chierici, cavalieri e religiosi vari; agli stessi chierici si concedeva di circondare con un muro chiese e case claustrali senza alcuna contraddizione ${ }^{93}$. A Parma, nel 1255, si stabilivano regole tra vicini che avessero muri in comune e androni privati, tra cui la possibilità di edificare, sopra gli androni, portici o porticelli ${ }^{94}$. A Siena, nel 1262 , chi avesse innalzato la casa iuxta le mura del comune era obbligato a costruirci merlos et pectorales, ma non veniva perseguito chi avesse addossato edifici sulle stesse mura dal tempo del podestà Ugone di Castello indietro ${ }^{95}$; inoltre si concedeva la possibilità di facere fundacum sive lembolum $\mathrm{e}$ realizzarne la porta nelle mura comunali al prezzo di 25 lire per porta; di costruire case, torri o altri edifici, in città o nei borghi, di qualunque altezza e ampiezza, tranne quando la maggioranza del consiglio proponesse la riduzione di determinati edifici ad una certa misura; di edificare all'interno di una torre di cui si possedesse una quota, senza contraddizione da parte dei consoci ${ }^{96}$.

Anche nell'ambito dell'edilizia privata, pertanto, si nota una chiara tendenza di crescita delle capacità di intervento delle autorità urbane di pari passo con l'evoluzione dei regimi politici e l'ascesa dei gruppi popolari. Tuttavia, soprattutto alcune grandi città ma-

89 «Liber statutorum comunis novocomi». In Historiae patriae monumenta edita iussu Regis Karoli Alberti, tomus XVI, Leges municipales, tomus secundus. Aosta: Fratelli Bocca, 1838, p. 153; Fiumi, Statuti di Volterra, pp. 160-162; Liberali, Gli statuti, pp. 247, 261 e 288; Adriani, Statuti, pp. 246-247 e 311-312; Gabotto, Gli statuti di Biella, p. 357; Frati, Statuti di Bologna, pp. 233-235, 280, 389 e 401; Banchi, Breve degli officiali, pp. 57-67, 86-88 e 93-94; Zdekauer, Il costituto, pp. 293 e 307; Cerlini, Consuetudini, p. 222; Ceci e Pensi, Statuto di Todi, pp. 55-56; Ceruti, Statuta communitatis Novariae, pp. 183-184; Bartoli Langeli e Caprioli, Statuto, pp. 195 e 213-214; Bonaini, Statuti inediti, p. 517.

90 Adriani, Statuti, pp. 147 e 246; Banchi, Breve degli officiali, pp. 57-67, 86-88; Cerlini, Consuetudini, p. 236.

91 Ambienti che conducevano dall'accesso dell'edificio all'area esterna.

92 Liberali, Gli statuti, p. 266.

93 «Statuta et privilegia civitati Augustae Praetoriae». In Historiae patriae monumenta edita iussu Regis Karoli Alberti, tomus II, Leges municipales. Aosta: Tipografia Regia, 1838, p. 34.

94 Ronchini, Statuta communis Parmae, pp. 254-255.

${ }_{95}$ Zdekauer, Il costituto, pp. 276-277. Chi avesse addossato edifici alle mura cittadine non era perseguito nemmeno a Todi: Ceci e Pensi, Statuto di Todi, p. 96.

96 Zdekauer, Il costituto, pp. 286 e 403. 
rittime, per lo meno stando alle fonti conservate, anticiparono tale cronologia: quando altrove il pubblico non era ancora in grado di dettare capillari regolamentazioni edilizie, né avvertiva forti necessità ideologiche e di sviluppo urbanistico in tal senso, a Genova e Pisa - comunità urbane dallo sviluppo economico precoce — si determinava l'esigenza politica di gestire un'impressionante edilizia privata, che peraltro contribuiva alla conflittualità tra le famiglie eminenti.

\subsection{Regolamentazioni su igiene e sicurezza}

Le politiche atte al miglioramento delle condizioni di vita collettive, anche in ambito urbanistico, rappresentano - come già spiegato nel caso delle reti viarie - un aspetto caratteristico dell'evoluzione duecentesca dei regimi cittadini e dell'ascesa dei gruppi popolari ${ }^{97}$. Numerose rubriche degli statuti del secolo xIII mostrano che i governi delle città fossero molto sensibili ai temi della salute e della sicurezza pubbliche, segno di grande maturazione politica.

A proposito di condizioni igieniche, dunque, prima del Duecento «i secchiai e i servizi igienici delle case scaricavano i liquami per caduta libera negli spazi laterali che separavano una casa dall'altra ${ }^{98}$, alla cui pulizia dovevano provvedere gli stessi abitanti delle case. In seguito tali spazi iniziarono ad essere vietati e apparve la canalizzazione «che correva al centro degli isolati, parallela alle facciate e tangente le aree cortilive interne, sul confine dei singoli lotti, che serviva per raccogliere le acque nere domestiche»" ${ }^{99}$. Al di là delle questioni tecniche, alcuni esempi tipici di normative in favore dell'igiene e contro l'inquinamento provengono da Biella (1245), dove si vietava di convogliare sozzure, putredine e acque sporche nelle vie pubbliche e nella rugia, la canalizzazione artificiale costruita per l'approvvigionamento idrico ${ }^{100}$. A Bologna, nel 1250, una rubrica statutaria recitava: Ad removendas putredines civitatis, statuimus et ordinamus quod androna sive coacla, que est jnter domos illorum de valdoniga et viam medij, debeat esse aperta et salegata ${ }^{101}$. Altre norme di simile tenore sono riscontrabili nelle statuizioni di Como (1221), Verona (1228), Vercelli (1241), Siena (1250) e Novara (1277) ${ }^{102}$. Particolarmente interessante una rubrica del 1265 proveniente da Reggio Emilia, che per una serie di vie ben specificate disponeva la distruzione di enxutas vel aquarolos posti sopra i canali e le vie stesse, la chiusura delle plazolae site in quelle aree, per mezzo di muri ampi in longitudine 4 braccia, e la copertura dei dugalia plazolarum per una lunghezza pari a quella dei portici, per evitare che la putredine investisse quei percorsi ${ }^{103}$.

${ }^{7}$ Si vedano Bocchi, La modernizzazione», pp. 331-333 e Menant, L'Italia dei comuni, pp. 162-163.

98 Bocchi, La modernizzazione», p. 334.

99 Bocchi, La modernizzazione", p. 335.

100 Gabotto, Gli statuti di Biella, pp. 357-398.

101 Frati, Statuti di Bologna, p. 449.

102 Liber statutorum comunis novocomi, p. 155; Liber iuris civilis urbis Veronae, pp. 125-127; Adriani, Statuti, pp. 150-153; Banchi, Breve degli officiali, pp. 86-88; Ceruti, Statuta communitatis Novariae, pp. 8-9.

103 Cerlini, Consuetudini, pp. 251-253. 
A proposito di sicurezza, poi, la preoccupazione principale delle autorità cittadine era relativa agli incendi, che rappresentavano il pericolo più grande. La lotta agli incendi prevedeva soprattutto regolamentazioni incentrate sui materiali per le costruzioni, con particolare riferimento ai tetti degli edifici: si doveva infatti evitare che venissero usati materiali infiammabili per le coperture di case e altri fabbricati, come pure porre mucchi di paglia, fieno, strame e frasche nei pressi delle abitazioni o sui solaria delle stesse. Inoltre si doveva vietare il trasporto di fiamme non protette, il lavoro notturno su elementi infiammabili (come la canapa) e si dovevano allontanare dai centri cittadini quei mestieri che utilizzassero forni e altri strumenti basati sul fuoco ${ }^{104}$. Specialmente nel corso del Duecento molte città inserirono nelle proprie statuizioni norme in tal senso: è il caso, ad esempio, di Pavia (1192-1206), Como (1209), Biella (1245) e Bassano del Grappa $(1259)^{105}$. Particolarmente interessante la testimonianza proveniente da Siena, dove nel costituto del 1262 si stabiliva che la volta della zecca fosse di muratura, proprio per il timore che da essa si propagassero incendi ${ }^{106}$. Infine una rubrica degli statuti bolognesi del 1250 ordinava l'arretramento di un metro delle grondaie delle case poste in una specifica contrada, dal momento che questi elementi sporgevano così tanto dai due lati della strada che quasi arrivavano a toccarsi, con grande pericolo in caso di fiamme ${ }^{107}$.

In questo genere di politiche, inoltre, il formulario delle norme si somiglia in numerose città. Ovunque si incontrano rubriche che riportano passaggi come: de non prohiciendis turpitudinibus in vias; de letamine et scopaturis et aliis inmundis in viis vel rugiis non prohiciendis; quod nullus debeat ponere fenum, paleas, fraschas neque facere ramatas frascharum super stratam nec super solarium; quod nemo debeat tenere nec facere domos paleatas e removeri facere omnia tecta de palea. Segno, oltre che della maturità cui si accennava, anche di una certa competizione tra le diverse realtà urbane.

\section{OSSERVAZIONI CONCLUSIVE}

L'analisi sviluppata - che ha come fonte privilegiata gli statuti cittadini editi - ha mostrato da una parte il ruolo crescente dei governi locali come soggetti produttori di grandi edifici pubblici, nei quali si univano utilità funzionale e valore identitario. D'altra parte, meno eclatante ma evidentissima nelle redazioni statutarie, risulta l'attività regolativa degli stessi governi urbani: un groviglio di norme e disposizioni, infatti, mirava a disciplinare l'enorme crescita dell'edilizia privata nelle città italiane del Duecento. Il fine, anche in questo caso, era l'utile, il bene di tutti. Un concetto che, come detto, penetrò gradualmente nella mentalità comunale soprattutto per effetto delle svolte ricordate: la pace di Costanza, il passaggio dai regimi consolari a quelli podestarile e, ancor più, la

104 Si vedano Balestracci, Duccio. «La lotta contro il fuoco (xII-XVI secolo)». In Cristiani, Emilio e Salvatori, Enrica. Città e servizi sociali nell'Italia dei secoli XII-XV. Atti del $12^{\circ}$ Convegno internazionale di studi. Roma: Viella, 1990, pp. 417-438 e Menant, L'Italia dei comuni, pp. 165-166.

105 Soriga, Il memoriale, p. 113; Liber statutorum comunis novocomi, p. 159; Gabotto, Gli statuti di Biella, pp. 354 e 393; Fasoli, Statuti del comune di Bassano, p. 67.

106 Zdekauer, Il costituto, p. 126.

107 Frati, Statuti di Bologna, p. 403. 
crescita del ruolo politico dei gruppi popolari. Tali eventi determinarono un consistente incremento anche della redazione degli statuti, in particolare nelle città in cui il Popolo raggiunse i vertici del governo. Promulgazione di nuovi statuti e tema dell'utile, o del bene comune, si configurano dunque entrambi come risultati dell'evoluzione dei regimi cittadini verso una maggiore consapevolezza del proprio ruolo pubblico. Quando gli eventi ricordati davano di volta in volta un nuovo impulso a tale processo evolutivo, allo stesso tempo ciò si rifletteva sulla moltiplicazione delle norme e sull'accentuazione nelle stesse, anche in campo edilizio e urbanistico, della sensibilità per alcune tematiche: una maggiore attenzione per la tutela degli spazi pubblici, dell'igiene, della sicurezza dei cittadini, così come una maggiore attenzione per la regolamentazione dell'edilizia privata. La sensibilità, cioè, per la creazione di contesti cittadini più adeguati a rispondere alle esigenze della vita quotidiana della comunità in rapporto alla materia urbanistica, che si rivelò particolarmente evidente durante la fase popolare. L'esempio principale è Bologna, dove tra $1245 \mathrm{e}$ 1267 le statuizioni promulgate furono vaste, numerose e fortemente intrise di questo genere di preoccupazioni, come le analisi riportate nelle pagine precedenti hanno mostrato. Tuttavia non deve essere dimenticato che in questa capillare opera di regolamentazione i regimi popolari affermavano sé stessi e il proprio diritto di controllare lo spazio urbano. In altre parole, anche nella regolamentazione minuta dell'edilizia privata, i governi del Popolo esprimevano in maniera incontrovertibile il loro ruolo di attori pubblici.

Quanto al tema della circolazione dei modelli statutari da una città all'altra, anche per il settore urbanistico, essa accentuò il suo passo soprattutto a partire dalla professionalizzazione dell'ufficio podestarile e dalla circolazione di questi professionisti della politica. Oltre ai casi già evidenziati precedentemente, che sembrano mostrare come gli influssi documentari si riflettessero soprattutto in alcuni ambiti delle statuizioni, altrettanto interessante risulta una rubrica degli statuti di Novara del 1277, che regolamentava una serie di divieti edilizi riguardanti le mura cittadine, per la preservazione delle stesse: essa riprendeva una norma emanata da un podestà attivo nei primi decenni del secolo XIII, Rubaconte Mandelli, noto come podestà "edificatore" anche in altre realtà urbane, ad esempio Firenze, dove aveva fatto costruire il ponte omonimo e pavimentare le strade $^{108}$. Non è stato invece possibile notare significative differenze, negli orientamenti politici edilizi e urbanistici, tra città demograficamente maggiori e minori. Nella netta gran parte dei centri urbani esaminati, grandi, medi o piccoli che fossero, i processi di evoluzione istituzionale portarono un po' ovunque le sensibilità precedentemente tirate in ballo a ritrovarsi al centro delle attenzioni politiche, anche in considerazione di un tema già affrontato, quello della circolazione degli ufficiali e dei modelli documentari. 
Adriani, Giovan Battista. Statuti del comune di Vercelli dell'anno 1241. Torino: Paravia e comp., 1877. Andenna, Giancarlo. «Il monastero e l'evoluzione urbanistica di Brescia tra XI e XII secolo». In $S$. Giulia di Brescia. Archeologia, arte, storia di un monastero regio dai Longobardi al Barbarossa. Atti del Convegno. Brescia: Grafo, 1992, pp. 93-118.

"Antiquae collationes statuti veteris civitatis Pergami». In Historiae patriae monumenta edita iussu Regis Karoli Alberti, tomus XVI, Leges municipales, tomus secundus, pars altera. Aosta: Fratelli Bocca, 1876, pp. 1921-2046.

Balestracci, Duccio. «Immigrazione e morfologia urbana nella Toscana bassomedievale». In Maire Vigueur, Jean-Claude. D'une ville à l'autre. Structures matérielles et organisation de l'espace dans les villes européennes (XIIIe - XVIe siècle). Actes du colloque organisé par l'École Française de Rome. Paris: École Française de Rome, 1989, pp. 87-105.

Balestracci, Duccio. «La lotta contro il fuoco (XII-XVI secolo)». In Cristiani, Emilio e Salvatori, Enrica. Città e servizi sociali nell'Italia dei secoli XII-XV. Atti del $12^{\circ}$ Convegno internazionale di studi. Roma: Viella, 1990, pp. 417-438.

Balestracci, Duccio. «La politica delle acque urbane nell'Italia comunale». Mélanges de l'École Française de Rome, 1992, vol. 104, pp. 431-479.

Balestracci, Duccio e Piccinni, Gabriella. Siena nel Trecento. Assetto urbano e strutture edilizie. Firenze: Nuova Grafica Fiorentina, 1977.

Banchi, Luciano. «Breve degli officiali del comune di Siena compilato nell'anno 1250». Archivio Storico Italiano, 1866, serie terza, tomo III, parte II, pp. 3-104.

Banti, Ottavio. I brevi dei consoli del comune di Pisa degli anni 1162 e 1164. Roma: Tipografia del Senato, 1997.

Bargagli Petrucci, Fabio. Le fonti di Siena e i loro acquedotti. Siena-Firenze-Roma: Olschki, 1906.

Bartoli Langeli, Attilio e Caprioli, Severino. Statuto del comune di Perugia del 1279. Perugia: Deputazione di Storia Patria per l'Umbria, 1996.

Berlan, Francesco. Statuti di Pistoia del secolo XII. Bologna: Romagnoli, 1882.

Besta, Enrico e Barni, Gian Luigi. Liber consuetudinum Mediolani anni MCCXVI. Milano: Giuffrè, 1949.

Bocchi, Francesca. Bologna nei secoli IV-XIV. Mille anni di storia urbanistica di una metropoli medievale. Bologna: Bononia University Press, 2008.

Bocchi, Francesca. «La modernizzazione delle città medievali». In La costruzione della città comunale italiana (secoli XII-inizio XIV). Atti del convegno. Pistoia: Centro Italiano di Storia e Arte, 2009, pp. 333-347.

Bonaini, Francesco. Statuti inediti della città di Pisa dal XII al XIV secolo. Firenze: Vieusseux, 1854, vol. I.

Bortolami, Sante. «Acque, mulini e folloni nella formazione del paesaggio urbano medievale (secoli XI-XIV)». In Paesaggi urbani dell'Italia padana nei secoli VIII-XIV. Bologna: Cappelli, 1988, pp. 277-330.

Calleri, Marta. «I più antichi statuti di Savona». Atti della società ligure di storia patria, 1997, nuova serie, vol. 37, n. ${ }^{\circ}$ II, pp. 117-212.

Carocci, Sandro. «Baroni in città. Considerazioni sull'insediamento e i diritti urbani della grande nobiltà». In Rome aux XIIIe et XIVe siècles. Cinq études réunies par Étienne Hubert. Roma: École Française de Rome-Viella, 1993, pp. 137-173.

Ceci, Getulio e Pensi, Giulio. Statuto di Todi del 1275. Todi: Trombetti, 1897.

Cerlini, Aldo. Consuetudini e statuti reggiani del secolo XIII. Milano: Hoepli, 1933. 
Ceruti, Antonio. Statuta communitatis Novariae anno 1277. Novara: Fratelli Miglio, 1878.

Conti, Mario Niccolò. Corpus statutorum lunigianensium. La Spezia: Accademia mLunigianese di Scienze, 1979, vol. I.

Crouzet-Pavan, Élisabeth. «Sopra le acque salse»: espaces urbains, pouvoir et société à Venise à la fin du Moyen Âge. Paris \& Roma: École Française de Rome, 1992.

Crouzet-Pavan, Elisabeth. "La cité communale en quête d'elle-même: la fabrique des grands espaces publics». In La costruzione della città comunale italiana (secoli XII-inizio XIV). Atti del convegno. Pistoia: Centro Italiano di Storia e Arte, 2009, pp. 91-130.

De Giovanni, Cesarina. "L'ampliamento di Assisi nel 1316». Bollettino della Deputazione di Storia Patria per l'Umbria, 1975, vol. LXXII, n. ${ }^{\circ}$ I, pp. 1-78.

De Minicis, Elisabetta. Case e torri medievali I. Atti del Convegno La città e le case: tessuti urbani, domus e case-torri nell'Italia comunale. Roma: Edizioni Kappa, 1996.

De Minicis, Elisabetta e Guidoni, Enrico. Case e torri medievali II. Atti del Convegno La città, le torri e le case: indagine sui centri dell'Italia comunale. Roma: Edizioni Kappa, 2001.

De Minicis, Elisabetta e Guidoni, Enrico. Case e torri medievali III, Atti del Convegno Case e torri medievali: indagine sui centri dell'Italia comunale. Roma: Edizioni Kappa, 2005.

Diacciati, Silvia e Tanzini, Lorenzo. «Uno spazio per il potere: palazzi pubblici nell'Italia comunale». In Diacciati, Silvia e Tanzini, Lorenzo (eds.). Società e poteri nell'Italia medievale. Studi degli allievi per Jean-Claude Maire Vigueur. Roma: Viella, 2014, pp. 59-80.

Fanelli, Giovanni. Firenze. Bari: Laterza, 1980.

Fasoli, Gina. Statuti del comune di Bassano dell'anno 1259 e dell'anno 1295. Venezia: Regia Deputazione di Storia Patria per le Venezie, 1940.

Federici, Vincenzo. Statuti della provincia romana. Roma: Tipografia del Senato, 1930, vol. II.

Fiumi, Enrico. Statuti di Volterra, I, (1210-1224). Firenze: Deputazione di storia patria per la Toscana, 1951.

Franceschi, Franco. «I paesaggi della produzione». In La costruzione della città comunale italiana (secoli XII-inizio XIV). Atti del convegno. Pistoia: Centro Italiano di Storia e Arte, 2009, pp. 167-194.

Frati, Luigi. Statuti di Bologna dall'anno 1245 all'anno 1267, II. Bologna: Regia Tipografia, 1876.

Gabotto, Ferdinando. «Gli statuti di Biella secondo il codice originale del 1245». Biblioteca della società storica subalpina, 1908, vol. 34, pp. 363-364.

Grillo, Paolo. Milano in età comunale (1183-1276). Istituzioni, società, economia. Spoleto: Fondazione Centro Italiano di Studi sull'Alto Medioevo, 2001.

Grossi Bianchi, Luciano e Poleggi, Ennio. Una città portuale nel medioevo: Genova nei secoli X-XVI. Genova: Sagep, 1980.

Guglielmotti, Paola. Genova. Spoleto: Fondazione Centro Italiano di Studi sull'Alto Medioevo, 2013.

Heers, Jacques. «Urbanisme et structure sociale à Gênes au Moyen Âge». In Heers, Jacques. Société et économie à Gênes (XIVe-XVe siècles). Londra: Variorum, 1979, pp. 371-412.

Heers, Jacques. Espaces publics, espaces privés dans la ville. Le Liber terminorum de Bologne 1294. Paris: Editions du centre national de la recherche scientifique, 1984.

Heers, Jacques. «Les villes d'Italie centrale et l'urbanisme: origines et affirmation d'une politique (environ 1200-1350)». Mélanges de l'École française de Rome, 1989, vol. 101, n.o 1, pp. 67-93.

Hubert, Étienne. Espace urbain et habitat à Rome du Xe siècle à la fin du XIIIe siècle. Roma: École Française de Rome, 1990.

Hubert, Étienne. «Propriété ecclésiastique et croissance urbaine: à propos de l'Italie centroseptentrionale, XIIe-début du xIve siècle». In Gli spazi economici della chiesa nell'occidente 
mediterraneo (secoli XII-metà XIV). Atti del convegno. Pistoia: Centro Italiano di Storia e d'Arte, 1999 , pp. 125-155.

Hubert, Étienne. «La construction de la ville. Sur l'urbanisation dans l'Italie médiévale». Annales. Histoire, Sciences Sociales, 2004/1 (59e année), pp. 109-139.

Hubert, Étienne. «Urbanizzazione, immigrazione e cittadinanza (xII-metà XIV secolo). Alcune considerazioni generali». In La costruzione della città comunale italiana (secoli XII-inizio XIV). Atti del convegno. Pistoia: Centro Italiano di Storia e Arte, 2009, pp. 131-145.

Hyde, John Kenneth. Padova nell'età di Dante. Storia sociale di una città-stato italiana. Trieste: LINT, 1985.

Imperiale, Cesare. Codice diplomatico della Repubblica di Genova. Roma: Tipografia del Senato, 1936.

Lazzarini, Isabella e Menant, François. «Les podestats». In Caciorgna, Maria Teresa, Carocci, Sandro e Zorzi, Andrea (eds.). I comuni di Jean-Claude Maire Vigueur. Percorsi storiografici. Roma: Viella, 2014, pp. 177-199.

Liber iuris civilis urbis Veronae, per Bartholomaeum Campagnolam. Verona: apud Petrum Antonium Bernum, 1728.

«Liber statutorum comunis novocomi». In Historiae patriae monumenta edita iussu Regis Karoli Alberti, tomus XVI, Leges municipales, tomus secundus. Aosta: Fratelli Bocca, 1838, pp. 133-258.

Liberali, Giuseppe. Gli statuti del comune di Treviso. Venezia: Regia Deputazione di Storia Patria per le Venezie, 1950.

Lomastro, Francesca. Spazio urbano e potere politico a Vicenza nel XIII secolo. Vicenza: Accademia Olimpica, 1981.

Maire Vigueur, Jean-Claude. «L'essor urbain dans l'Italie médiévale: aspects et modalités de la croissance». In Europa en los umbrales de la crisis (1250-1350). XXI Semana de estudios medievales de Estella. Pamplona: Gobierno de Navarra, 1996, pp. 171-204.

Maire Vigueur, Jean-Claude. «La politique monumentale des communes et des seigneuries: un essai de comparaison (Italie centrale, XIV siècle)». In Albonico, Simone e Romano, Serena (eds.). Courts and courtly cultures in early modern Italy and Europe. Roma: Viella, 2016, pp. 37-66.

Menant, François. L'Italia dei comuni (1100-1350). Roma: Viella, 2011.

Menzinger, Sara. «Mura e identità civica in Italia e in Francia meridionale (secc. XII-XIV)». In Menzinger, Sara (ed.). Cittadinanze medievali: dinamiche di appartenenza a un corpo comunitario. Roma: Viella, 2017, pp. 65-111.

Milani, Giuliano. Bologna. Spoleto: Fondazione Centro Italiano di Studi sull'Alto Medioevo, 2012.

Milani, Giuliano. «Contro il comune dei milites. Trent'anni di dibattiti sui regimi di Popolo». In In Caciorgna, Maria Teresa, Carocci, Sandro e Zorzi, Andrea (eds.). I comuni di Jean-Claude Maire Vigueur. Percorsi storiografici. Roma: Viella, 2014, pp. 235-258.

Moretti, Italo. «I palazzi pubblici». In La costruzione della città comunale italiana (secoli XII-inizio XIV). Atti del convegno. Pistoia: Centro Italiano di Storia e Arte, 2009, pp. 67-80.

Mucciarelli, Roberta. «Demolizioni punitive: guasti in città». In La costruzione della città comunale italiana (secoli XII-inizio XIV). Atti del convegno. Pistoia: Centro Italiano di Storia e Arte, 2009, pp. 293-330.

Niccolai, Franco. Contributo allo studio dei più antichi brevi della Compagna genovese. Milano: Giuffrè, 1939.

Nicolini, Ugolino. "Le mura medievali di Perugia». In Storia e arte in Umbria nell'età comunale. Atti del convegno. Perugia: Universitá degli Studi di Perugia, 1971, pp. 695-769. 
Orlando, Ermanno. Venezia. Spoleto: Fondazione Centro Italiano di Studi sull'Alto Medioevo, 2016.

Pirani, Francesco. Fermo. Spoleto: Fondazione Centro Italiano di Studi sull'Alto Medioevo, 2010.

Riccetti, Lucio. La città costruita. Lavori pubblici e immagine in Orvieto medievale. Firenze: Le Lettere, 1992.

Ronchini, Amadio. Statuta communis Parmae digesta anno 1255. Parma: Officina Fiaccadori, 1856.

Settia, Aldo Angelo. "Lo sviluppo di un modello: origine e funzioni delle torri private urbane nell'Italia centrosettentrionale». In Paesaggi urbani dell'Italia padana nei secoli VIII-XIV. Bologna: Cappelli, 1988, pp. 155-171.

Settia, Aldo Angelo. «Cerchie murarie e torri private urbane». In La costruzione della città comunale italiana (secoli XII-inizio XIV). Atti del convegno. Pistoia: Centro Italiano di Storia e Arte, 2009, pp. 45-66.

Silvestrelli, Maria Rita. "L'edilizia pubblica del comune di Perugia: dal palatium comunis al palatium novum populi». In Società e istituzioni dell'Italia comunale: l'esempio di Perugia (secoli XII-XIV). Perugia: Deputazione di Storia Patria per l'Umbria, 1988, vol. II, pp. 482-604.

Silvestrelli, Maria Rita. "Grandi cantieri e palazzi pubblici. L’esempio di Perugia». In CrouzetPavan, Élisabeth (ed.). Pouvoir et édilité: les grands chantier dans l'Italie communale et seigneuriale. Roma: École Française de Rome, 2003, pp. 105-158.

Soldi Rondinini, Gigliola. «Evoluzione politico-sociale e forme urbanistiche nella Padania dei secoli XII-XIII: i palazzi pubblici». In La pace di Costanza 1183. Un difficile equilibrio di poteri fra società italiana ed impero. Convegno Internazionale. Bologna: Cappelli, 1984, pp. 85-98.

Solmi, Arrigo. «Le leggi più antiche del comune di Piacenza». Archivio Storico Italiano, 1915, vol. 75, pp. 65-80.

Soriga, Renato. «ll memoriale dei consoli del comune di Pavia». Bollettino della Società Pavese di Storia Patria, 1913, vol. 13, pp. 103-118.

«Statuta et privilegia civitati Augustae Praetoriae». In Historiae patriae monumenta edita iussu Regis Karoli Alberti, tomus II, Leges municipales. Aosta: Tipografia Regia, 1838, pp. 33-38.

Szabó, Thomas. «Il controllo dello spazio e la genesi della rete viaria comunale nel Medioevo». In Rossetti, Gabriella (ed.). Spazio, società, potere nell'Italia dei comuni. Napoli: Liguori, 1986, pp. 27-36.

Szabó, Thomas. «Genesi e sviluppo della viabilità urbana». In La costruzione della città comunale italiana (secoli XII-inizio XIV). Atti del convegno. Pistoia: Centro Italiano di Storia e Arte, 2009, pp. 149-162.

Sznura, Franek. L'espansione urbana di Firenze nel Dugento. Scandicci: La Nuova Italia, 1975.

Tanzini, Lorenzo. Firenze. Spoleto: Fondazione Centro Italiano di Studi sull'Alto Medioevo, 2016.

Varanini, Gian Maria. «L'espansione urbana di Verona in età comunale: dati e problemi». In Rossetti, Gabriella (ed.). Spazio, società, potere nell'Italia dei comuni. Napoli: Liguori, 1986, pp. $1-25$.

Varanini, Gian Maria. «Torri e casetorri a Verona in età comunale: assetto urbano e classe dirigente». In Paesaggi urbani dell'Italia padana nei secoli VIII-XIV. Bologna: Cappelli, 1988, pp. 173-249.

Vignoli, Paola. I costituti della legge e dell'uso di Pisa (sec. XII). Roma: Tipografia del Senato, 1997. Yunn, Amee. The Bargello: a new history of the first communal palace of Florence, 1255-1346. New York: New York University, 2009.

Zdekauer, Lodovico. Il costituto del comune di Siena dell'anno 1262. Milano: Hoepli, 1897. 